\title{
La retirada deportiva en deportes colectivos: comparativa profesionales y amateurs
}

\section{Sport retirement among team sports: professional and amateur comparative}

\author{
Cristina López de Subijana Hernández ${ }^{1 *}$, Maribel Barriopedro Moro ${ }^{1}$, Carlos Alberto Muniesa ${ }^{2}$ y Jose Luis Galocha ${ }^{1}$ \\ 1 Dpto. de Ciencias Sociales de la Actividad Física, del Deporte y del Ocio. Facultad de Ciencias de la Actividad Física y del Deporte-INEF, Universidad \\ Politécnica de Madrid. \\ 2 Facultad de Ciencias de la Actividad Física y del Deporte, Universidad Europea de Madrid
}

Resumen: En los últimos tiempos han aumentado el interés por la vida de los deportistas tras su carrera en la élite (Park, Lavalle,y Tod 2013).La propia idiosincrasia económica de cada deporte hace que sean interesantes las comparaciones entre diferentes grupos (Aquilina, 2013).Los objetivos de este estudio fueron analizar si la calidad de la retirada deportiva, evaluada a partir de las dificultades percibidas en diferentes ámbitos, y las condiciones asociadas a la misma (planificación, voluntariedad, tipo de carrera deportiva y nivel de estudios) era diferente para los deportistas profesionales y amateurs. Se utilizó un estudio descriptivo transversal mediante encuestas, con muestreo intencional, administrando un cuestionario de preguntas cerradas, elaborado ad hoc. Participaron un total de 127 deportistas, de ellos 53 profesionales y 74 no profesionales. Los deportistas profesionales experimentaron en mayor grado dificultades relacionadas con su carrera profesional, con su familia, con su red social y vivieron su proceso de integración laboral de una forma menos positiva que los deportistas amateurs. Estos últimos, experimentaron en mayor grado dificultades económicas. Como cabría esperar debido a un vínculo más fuerte con el deporte, un mayor número de deportistas profesionales se dedicó exclusivamente al deporte, mientras que un mayor número de deportistas amateurs compaginaron deporte y trabajo. Además, entre aquellos que compatibilizaron el deporte con los estudios, más deportistas profesionales priorizaron el deporte. La planificación de la retirada fue más frecuente entre los profesionales y no se observaron diferencias en relación a la voluntariedad de la retirada ni en el nivel de estudios.

Palabras Clave: deporte de élite; carrera dual, formación, ingresos,
Abstract: Recently researchers have increased their interest in the post sport life of the elite athletes (Park, Lavalle,and Tod 2013). The unique economic features of each sport make interesting the comparison between them (Aquilina, 2013).The aims of this study were to analyse if the quality of the sport retirement (based on the perceived difficulties on different dimensions), the features of the sport retirement (planning, the willfulness, the type of career path and the academic level) were different between professional and amateur athletes. A cross-sectional descriptive study based on surveys was applied. An ad hoc questionnaire of closed question was answered by 127 retired elite athletes. Fifty-three athletes were from a professional sport while seventy-four athletes were from a non-professional sport. The professional athletes experienced in a greater grade difficulties related with their working career, their family, their social network, and they lived the working integration less positively than the amateur athletes. The amateur athletes suffered more frequently economic difficulties. As it could be expected due the greater relation with sport, a higher number of professional athletes were exclusively devoted to sport, while more amateur sport combined their sport career with working. Moreover, among those who combined the sport career with studies, the professional athletes gave more frequently priority to sport. Planning the retirement was more frequent among the professional athletes. No significant differences were found in relation with the willfulness of the sport retirement and the study level of the athletes.

Keywords: elite sport; dual career; education, earnings.

\section{Introducción}

Los primeros estudios sobre el desarrollo de la carrera profesional tras la retirada deportiva, comenzaron a realizarse a finales de los años 70 y ponían de manifiesto que los deportistas no presentaban ninguna desventaja en cuanto al desarrollo de su vida profesional comparados con la población general (Conzelmann, y Nagel, 2003). No obstante, estos estudios están realizados sobre deportistas que compitieron en una época en la que el volumen de entrenamiento y la frecuencia de competiciones era mucho menor. Algunos

Dirección para correspondencia [Correspodence address]: Cristina López de Subijana. Dpto. de Ciencias Sociales de la Actividad Física, del Deporte y del Ocio. Facultad de Ciencias de la Actividad Física y del Deporte-INEF, UPM. Av. Martín Fierro, 7. 28040 Madrid (Espańa) E-mail: c.lopezdesubijana@upm.es autores han señalado que la dedicación al alto rendimiento puede verse acompañada de dificultades académicas y profesionales (Brandao, y Viera, 2013; CecićErpič, Wylleman, y Zupančič, 2004; Cosh y Tully, 2014; Torregrosa, Sánchez, y Cruz, 2004). La Comisión Europea de Educación, Cultura y Deporte (2012), sensible a esta problemática, acuñó el término Carrera Dual (DC)para hacer referencia a la compatibilización del deporte con una segunda actividad vocacional (estudios o trabajo) y la promoción de esta CD, dados sus beneficios (Tekavc, Wylleman, y CecićErpić, 2015; Torregrosa, Ramis, Pallarés, Azócar, y Selva, 2015; Villanova, y Puig, 2014) es un aspecto central en los programas actuales de apoyo al deportista (Henry, 2013).

El estudio de la retirada deportiva se enmarca en un en- 
foque holístico (Wylleman, y Lavalle, 2004) que analiza la carrera del deportista desde cuatro dimensiones: la deportiva, la psicológica, la psicosocial y la académico-vocacional. En 2013, estos mismos autores añadieron la dimensión económica, como una quinta dimensión, basada en la fuente de ingresos del deportista (Wylleman, Reints, y De Knop, 2013). Este modelo holístico es una referencia general y aspectos concretos, como la cultura, la organización deportiva y el país en el que se desarrolla la carrera deportiva, hacen que sean necesarios modelos específicos (Battochio, Stambulova, y Schinke, 2015). Así mismo, la idiosincrasia de cada deporte (temporada de competiciones-viajes, formato de competiciones, posibilidades económicas, horas de entrenamiento requeridas, etc.) condicionan al deportista de cara a desarrollar una carrera más allá del deporte (Aquilina, 2013).

Siguiendo el Modelo Conceptual de la Adaptación de la retirada en los Deportistas de Elite, la calidad de la retirada depende de las razones de la finalización de la carrera deportiva, de los factores relacionados con la adaptación y de los recursos disponibles (Taylor \& Ogilvie, 1994; Taylor, Ogilvie, \& Lavallee, 2006). El fin de la carrera deportiva puede suponer una transición traumática. Algunos deportistas llegan a experimentar problemas de relaciones sociales, depresiones, adicciones al juego, alcohol y drogas (Stambulova, 2010). Estos problemas son más frecuentes entre aquellos deportistas que no planifican su retirada o se retiran de manera involuntaria (Stambulova, Alfermann, Statler y Côté, 2009).

Un número importante de deportistas, tanto profesionales como no profesionales, no son suficientemente conscientes de la importancia de desarrollar habilidades académicas o vocacionales de cara a su retirada (North y Lavallee, 2004; Samuel y Tenenbaum, 2011). Además, los programas de apoyo para deportistas profesionales se han introducido más tarde (Chambers, Gordon, y Morris, 2013) y con menor grado de estructuración y homogeneidad (Alesio, 2008). Por ejemplo, en la Liga de Futbol Australiano, se creó la figura del Gestor de Desarrollo del Jugador (Player Development Manager), cuya función no sólo es apoyar el desarrollo de la carrera deportiva, sino también orientar al deportista para su formación académica o vocacional y promover su interés fuera del ámbito del deporte profesional, con resultados positivos (Ryan, y Chambers, 2015; Pink, Saunders, y Stynes, 2014). Por el contrario, en Inglaterra, un programa de apoyo al deportista en el ámbito del Cricket profesional, no ha tenido resultados tan positivos (Roberts, Mullen, Evans, y Hall, 2015). Por otro lado, los deportistas profesionales comienzan a planificar su retirada en el último cuarto de sus carreras deportivas (Fortunato, y Marchant, 1999), lo que puede suponer una peor adaptación tras la retirada.

En Espańa, dentro del ámbito de los deportes colectivos, sólo hay dos ligas contempladas en la Ley de Deporte
10/90, como profesionales, en concreto la Liga Nacional de Fútbol Profesional (LFP) y la Asociación de clubes de Baloncesto (ACB), si bien es cierto que existen una serie ligas de deportes profesionalizados, en concreto la Asociación de Clubes Españoles de Balonmano, (ASOBAL) y la Liga Nacional de Fútbol Sala (LNFS) (Gamero, 2012), en las que la mayoría de sus deportistas dispone de vinculación laboral con sus respectivos clubes.

No todos los deportes tienen en su faceta profesional la máxima expresión del rendimiento deportivo de sus practicantes, pero si se dispone de un recurso objetivo para el reconocimiento del mismo, en concreto la relación de Deportistas de Alto Nivel (DAN). Los requisitos de acceso a esta condición se detallan en el Real Decreto 971 del año 2007. En el caso de que los deportistas que cumplen con dichos requisitos, éstos pasan a ser nombrados en el Boletín Oficial del Estado (BOE). Hecho que ocurre de manera trimestral.

El objetivo general de este estudio fue analizar si la calidad de la retirada deportiva es diferente para los deportistas profesionales y amateurs. Como objetivos específicos nos planteamos analizar si los deportistas profesionales y amateurs difieren en: i) las dificultades relativas a: su carrera profesional, estudios, familia, red social, ocio, salud y aspectos económicos; ii) las dificultades experimentadas para su integración laboral y iii) en las condiciones asociadas a la calidad de la retirada deportiva, en concreto: tipo de carrera deportiva, nivel de estudios y tipo de retirada.

\section{Método}

\section{Muestra}

La muestra estuvo integrada por un total de 127 sujetos, 53 jugadores profesionales y 74 jugadores de deportes no profesionales, todos ellos hombres. Para los deportistas profesionales, el criterio de inclusión en esta muestra fue haber participado en alguna liga profesional. En cuanto a los jugadores de deportes considerados no profesionales, el criterio de inclusión fue haber sido Deportista de Alto Nivel (DAN), en algún momento de su carrera deportiva.

Los deportes colectivos contemplados para los deportistas profesionales fueron: baloncesto $(n=19)$, balonmano $(n=20)$ y futbol sala $(n=14)$, mientras que los deportistas no profesionales procedían de hockey hierba $(n=12)$, rugby $(n=43)$ y

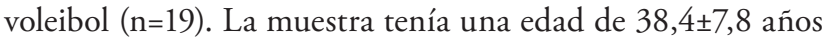
$(42,1 \pm 7,7$ los profesionales y $40 \pm 6,6$ años los amateurs) y llevaban $8,4 \pm 6,7$ años retirados $(8,8 \pm 7,3$ años los profesionales $y$ $7,6 \pm 5,3$ años los amateurs). En promedio semanalmente estos deportistas dedicaban $17 \pm 8$ a entrenar $(17,4 \pm 8$ horas los profesionales y $16,6 \pm 8,3$ horas los amateurs). 


\section{Instrumento}

Se desarrolló un cuestionario ad hoc, como técnica de recogida de datos, con 52 preguntas cerradas de opción múltiple, a partir de una adaptación del Athletics Retirement Questtionaire (Alfermann, Stambulova, y Zemaityte, 2004) y del Cuestionario de Integración Socio-Laboral para Deportistas de Élite (Barriopedro, Muniesa, y López de Subijana, 2016). Para este estudio, se tomaron las siguientes variables: edad, tiempo retirado, horas dedicadas al entrenamiento, nivel de estudios en la retirada, tipo de carrera deportiva, tipo de retirada (voluntaria/involuntaria; planificada/no planificada), grado en que sufrió dificultades relativas a: su carrera profesional, estudios, familia, red social, ocio, salud y aspectos económicos (escala tipo Likert de 1=Ninguno a 5=Mucho), cómo vivió su proceso de integración laboral (1=Muy mal a $6=$ Muy bien) y necesidad de un periodo de tiempo de adaptación tras la retirada.

\section{Procedimiento}

Se realizó un estudio descriptivo transversal mediante encuestas, con muestreo intencional. El cuestionario fue precedido de una carta de presentación, de forma que la contestación del mismo fuese voluntaria, se informaba del tratamiento de los datos y de la finalidad del estudio, siguiendo los requisitos éticos de la Declaración de Helsinky de 1975 y actualizada en octubre del 2000. El cuestionario fue administrado a través de correo electrónico vía Federaciones Deportivas Españolas y Asociaciones de Deportistas, para que éstas se lo reenviasen a los deportistas.

\section{Análisis de datos}

Para el análisis de resultados se realizaron pruebas $U$ de Mann Whitney, Chi-Cuadrado y t de Student. Como índice del tamaño del efecto se calculó eta cuadrado $\left(\eta^{2}=Z^{2} /(n 1+n 2)\right.$ para la prueba Ude Mann Whitney, el índice Phi $(\varphi)$ o la V de Cramer para la prueba Chi-Cuadrado y la d de Cohen para la prueba t.Se utilizaron los valores 0,$02 ; 0,13$ y 0,26 para interpretar el tamaño del efecto calculado a partir de $\eta^{2}$ como pequeño, mediano y grande, respectivamente, 0,10 ; 0,30 y 0,50para el índice $\varphi$ y la V de Cramer y 0,20; 0,50 y 0,80 para la d de Cohen(Cohen, 1988). El nivel de riesgo se estableció en 0,05 . Todos los análisis fueron realizados mediante el paquete estadístico SPSS, versión 18.0.

\section{Resultados}

En la Tabla 1 se presenta el grado en que los deportistas profesionales y amateurs percibieron dificultades en el momento de la retirada. Los deportistas profesionales percibieron en mayor grado dificultades relacionadas con su carrera profesional $\left(Z=1,88 ; p=.030 ; \eta^{2}=0,028\right)$, con la familia $(Z=2,78$; $\left.\mathrm{p}=.003 ; \eta^{2}=0,061\right)$ y con su red social $(Z=2,04 ; \mathrm{p}=.021$; $\eta 2=0,033)$ que los deportistas amateurs, si bien el tamaño de estas diferencias fue pequeño. Sin embargo, el grado de dificultades económicas percibidas por los deportistas profesionales fue menor que el percibido por los amateurs $(Z=2,02$; $\left.\mathrm{p}=.022 ; \eta^{2}=0,032\right)$.

Tabla 1. Grado en que sufrió diferentes dificultades en el momento de su retirada deportiva (1=Ninguno a $5=$ Mucho) para deportistas profesionales y amateurs. Media (M), Desviación Estándar (DE), estadístico de contraste para la prueba U de Mann-Whitney (Z), nivel de significación (p) y tamaño del efecto $\left(\eta^{2}\right)$.

\begin{tabular}{lccccccc}
\hline \multicolumn{7}{c}{ Profesionales } & \multicolumn{1}{c}{ Amateurs } \\
Dificultades & $\mathrm{M}$ & $\mathrm{DE}$ & $\mathrm{M}$ & $\mathrm{DE}$ & $\mathrm{Z}$ & $\mathrm{p}$ & $\eta^{2}$ \\
\hline Carrera Profesional & 2,0 & 1,1 & 1,7 & 1,2 & 1,88 &, 030 &, 028 \\
Estudios & 1,8 & 1,2 & 1,5 & 0,9 & 1,29 &, 086 & \\
Familia & 2,0 & 1,2 & 1,4 & 0,9 & 2,78 &, 003 &, 061 \\
Red Social & 1,9 & 1,2 & 1,6 & 1,0 & 2,04 &, 021 &, 033 \\
Ocio & 1,8 & 1,2 & 1,8 & 1,2 &, 08 &, 469 & \\
Salud & 1,9 & 1,1 & 1,9 & 1,1 &, 11 &, 456 & \\
Económicas & 2,4 & 1,3 & 1,9 & 1,3 & 2,02 &, 022 &, 032 \\
\hline
\end{tabular}

Tanto los deportistas profesionales como los amateurs vivieron su proceso de integración laboral de una forma bastante positiva, no obstante, los deportistas profesionales vivieron este proceso de integración laboral ( $1=$ Muy mal a $6=$ Muy bien) de una forma menos positiva $(4,2 \pm 1,5)$ que los deportistas amateurs $(4,6 \pm 1,6 ; Z=1,76 ; p=.036 ; \eta 2=0,024)$. De hecho, un porcentaje significativamente superior de deportistas profesionales $(56,5 \%)$ que de amateurs (26\%) informó de que necesitó un tiempo de adaptación tras la retirada $\left(\chi^{2}(1)=12,08\right.$; $\mathrm{p}=, 001 ; \varphi=0,31)$ aunque el tiempo que duró este periodo de adaptación fue significativamente menor entre los deportistas profesionales $(11,5 \pm 9,6$ meses) que entre los amateurs $(21,8$ $\pm 14,2$ meses; $\left.\mathrm{t}_{38}=2,75 ; \mathrm{p}=, 009 ; \mathrm{d}=0,49\right)$.

En relación al tipo de carrera deportiva (Tabla 2), la mayor parte de los deportistas realizaron una carrera dual (84,3\%). No obstante, hubo diferencias significativas de tamaño moderado entre los deportistas profesionales y amateurs $\left(\chi^{2}(3)=20,48 ; p<, 001 ; V=0,402\right)$. Un mayor número de deportistas profesionales $(20,8 \%)$ se dedicó exclusivamente al deporte, frente al $12,2 \%$ de los deportistas amateurs. Respecto a la compatibilización deporte y trabajo, hubo un mayor número de deportistas amateurs $(33,8 \%)$ que de profesionales $(9,4 \%)$. En cuanto a la compatibilización del deporte con los estudios, se podía optar por dar mayor prioridad al deporte o igual de prioritarios ambas carreras. Deportistas en carrera dual con estudios que priorizaron el deporte, hubo un mayor 
porcentaje de deportistas profesionales $(50,9 \%)$ que de amateurs $(20,3 \%)$. En cambio un mayor porcentaje de deportistas amateurs $(33,8 \%)$ que de profesionales $(18,9 \%)$ se decantaron por un modelo de carrera dual paralelo (dando la misma importancia a estudios y deporte).

Un 51,6\% de los deportistas planificó su retirada depor- tiva (Tabla 2), sin embargo, este porcentaje fue significativamente superior entre los deportistas profesionales (64\%) que entre los amateurs $\left(43,2 \% ; \chi^{2}(1)=5,15 ; \mathrm{p}=, 023 ; \varphi=0,204\right)$. El $85,5 \%$ de los deportistas se retiraron de manera voluntaria, y no se observaron diferencias significativas entre deportistas profesionales y amateurs $\left(\chi^{2}(1)=0,02 ; \mathrm{p}=, 893\right)$.

Tabla 2. Tipo de carrera deportiva, planificación y voluntariedad de la retirada para deportistas profesionales y amateurs.

\begin{tabular}{llccc}
\hline & & Profesionales & Amateurs & Total \\
\cline { 3 - 5 } & & $\%(\mathrm{~N}=53)$ & $\%(\mathrm{~N}=74)$ & $\%(\mathrm{~N}=127)$ \\
\hline \multirow{5}{*}{ Tipo de Carrera Deportiva } & Entrenar & 20,8 & 12,2 & 15,7 \\
& Deporte y Estudios, Prioridad Deporte & 50,9 & 20,3 & 33,1 \\
& Deporte y Estudios & 18,9 & 33,8 & 27,6 \\
& Deporte y Trabajo & 9,4 & 33,8 & 23,6 \\
Planificación Retirada & Si & 64,0 & 43,2 & 51,60 \\
& No & 36,0 & 56,8 & 48,40 \\
Voluntariedad Retirada & Si & 86,0 & 85,1 & 85,50 \\
& No & 14,0 & 14,9 & 14,50 \\
\hline
\end{tabular}

Un 63,8\% de los deportistas tenía estudios universitarios en el momento de la retirada (Tabla 3) y un 29,9\% tenía como máximo estudios secundarios. No se observaron diferencias significativas en el nivel de estudios alcanzado en el momento de la retirada entre los deportistas profesionales y amateurs $\left(\chi^{2}(5)=2,59 ; \mathrm{p}=, 763\right)$.

Tabla 3. Nivel de estudios en el momento de la retirada para deportistas profesionales y amateurs.

\begin{tabular}{lccc}
\hline & Profesionales & Amateurs & Total \\
\cline { 2 - 4 } & $\%(\mathrm{~N}=53)$ & $\%(\mathrm{~N}=74)$ & $\%(\mathrm{~N}=127)$ \\
\hline Primarios & 3,8 & 8,1 & 6,3 \\
Secundarios & 22,6 & 24,3 & 23,6 \\
Formación Profesional & 9,4 & 4,1 & 6,3 \\
Diplomatura & 18,9 & 16,2 & 17,3 \\
Grado & 37,7 & 40,5 & 39,40 \\
Tercer Ciclo & 7,5 & 6,8 & 7,10 \\
\hline
\end{tabular}

\section{Discusión}

Los objetivos de este estudio fueron, en primer lugar, analizar si la calidad de la retirada deportiva, evaluada a partir de las dificultades percibidas en diferentes ámbitos, era diferente para los deportistas profesionales y amateurs, y en segundo lugar, si estos deportistas se diferenciaban en las condiciones tradicionalmente asociadas a la calidad de la retirada deportiva, en concreto: planificación, voluntariedad, tipo de carrera deportiva y nivel de estudios.

Respecto a las dificultades experimentadas en la retirada, aunque el grado en que las sufrieron no fue elevado, como cabría esperar, los deportistas profesionales comparados con los amateurs, mostraron en menor grado dificultades económicas. Sin embargo, el grado en que los deportistas percibieron dificultades relacionadas con su carrera profesional, con su familia y con su red social fue mayor para los deportistas profesionales que para los amateurs. Además, los deportistas profesionalesvivieron su proceso de integración laboral de una forma menos positiva que los deportistas amateurs, sin embargo, el plazo de tiempo que necesitan para adaptarse a la nueva situación es menor (11,5 frente a 21,8 meses). Estos resultados son consistentes con los informados por Roberts et al. (2015) en jugadores de cricket profesional. La ausencia de responsabilidades durante la vida profesional de estos deportistas y la estructuración de sus vidas en esta etapa, hace que se encuentren con dificultades para adaptarse a una vida fuera del deporte (Lavallee, y Robinson, 2007).

Los resultados encontrados respecto al tipo de carrera deportiva son congruentes estudios previos. Es decir, el hecho de que en muchas ocasiones, a los deportistas profesionales, dado que disfrutan de un contrato económico, se les exige centrarse en su deporte y atender sólo de forma secundaria al desarrollo de su carrera fuera de este ámbito (Hickey, y Kelly, 2008). Son numerosos los estudios que indican que una carrera dual proporciona beneficios para la adaptación a la vida tras la retirada y por ello, constituye un aspecto central de los programas de apoyo al deportista (Henry, 2013). Los deportistas que siguen una carrera dual presentan en la retirada una mayor integración social, un mayor equilibrio en sus vidas y habitualmente consiguen un trabajo satisfactorio (Tekacv et al., 2015; Villanova, y Puig, 2014).La focalización 
en exclusiva en el deporte se ha asociado con características que dificultan esta transición, tales como la falta de planificación, una alta identidad deportiva, un menor apoyo social y una menor presencia de estrategias de afrontamiento (Torregrosa et al., 2015).

La planificación de la retirada, asociada a una menor frecuencia de dificultades para la adaptación a la vida tras el deporte (Stambulova et al., 2009), es más frecuente entre los profesionales que entre los amateurs. Sin embargo, algunos estudios indican que los deportistas profesionales que planifican lo hacen en una etapa tardía de su carrera deportiva (Fortunato, y Marchant, 1999). Este hecho alerta sobre la presencia de dificultades en la retirada. La mayor parte de los deportistas $(85,5 \%)$ se retiró de manera voluntaria, independientemente de que fueran profesionales o amateurs. Aunque la voluntariedad de la retirada se ha asociado a una mayor calidad en esta transición, cada vez hay un mayor acuerdo en que las razones para la retirada, con frecuencia, son múltiples y forman parte de un proceso dinámico de toma de decisiones (Alfermann, y Stambulova, 2007), siendo quizá más importante la buena disposición del deportista ante esta transición que el hecho de retirarse voluntariamente.

Por último, a pesar de que la carrera dual combinando estudios y deporte es más frecuente entre los deportistas profesionales, su nivel de estudios no es diferente del alcanzado por los deportistas amateurs. Posiblemente, este hecho se deba a que la mayor parte de los profesionales que combinan su deporte con los estudios priorizan el deporte. El hecho de que un 26,4\% de los deportistas profesionales sólo hubiera alcanzado estudios secundarios en la retirada, constituye un factor de alerta, ya que la falta de experiencia en el ámbito académico, constituye un factor que limita su habilidad para adaptarse al mundo laboral e incide en las dificultades experimentadas por estos deportistas para desarrollar su carrera profesional (Price, Morrison y Arnold, 2010; Roberts et al., 2015).

Este estudio presenta algunas limitaciones. La primera viene derivada de la variedad de deportes. Al tener diferentes deportes profesionales y amateurs, el contexto sociocultural varía en cada uno de ellos y es difícil hacer una aproximación a la situación de un conjunto de deportes. La segunda es la metodología utilizada para le recogida de datos, puesto que al ser tipo encuestas, habrá algunos deportistas que estén en peores condiciones que los participantes de este estudio, y por lo tanto los resultados pueden estar sesgados de forma positiva. Y la tercera limitación corresponde a la autoría de la respuesta, algo que con el fin de preservar el anonimato y al ser respondido online, no se pudo controlar.

\section{Conclusiones}

El hecho de que los deportistas profesionales perciban una remuneración, en muchos casos muy elevada, no garantiza que la transición a la vida fuera del deporte esté exenta de dificultades. Dados los riesgos que comportan estas carreras profesionales, cortas y desarrolladas en edades tempranas, los clubes no deberían eludir su responsabilidad social para con sus deportistas, justificándose con las remuneraciones que reciben y deberían contemplar la implementación de programas de apoyo. El análisis en profundidad de las dificultades que han de afrontar los deportistas profesionales en la retirada deportiva, teniendo en cuenta la especificidad de cada deporte, es necesario de cara a desarrollar programas efectivos de apoyo para su desarrollo psicológico, social, académico y vocacional.

\section{Agradecimientos}

Este proyecto ha sido financiado por el Centro de Estudios Olímpicos del Comité Olímpico Internacional, a través del Research Advanced Grant Program.

Este proyecto ha sido posible gracias a la financiación del Comité Olímpico Internacional.

\section{Referencias}

1. Alessio, S. (2008). Report on the recognition of the player development manager role in AFL: Prepared for the Australian Football League Players' Association.

2. Alessio, S. (2008). Report on the recognition of the player development manager role in AFL: Prepared for the Australian Football League Players' Association.(Issue Brief 1). Melbourne, VIC: AFL.

3. Alfermann, D., Stambulova, N., \& Zemaityte, A. (2004). Reactions to sport career termination: across-national comparison of German, Lithuanian, and Russian athletes. Psychology of Sport and Exercise, 5(1), 61-75.

4. Aquilina, D. (2013). A study of the relationship between elite athletes' educational development and sporting performance. The International Journal of the History of Sport, 30(4), 374-392.

5. Barriopedro, M., Muniesa, C., \&López de Subijana, C., (2016). Perspectiva de género en la Insercion laboral de los deportistas olímpicos españoles. [Gender perspective at theSpanish Olympic Athletes' employment]. Cuadernos de Psicología del Deporte, 16(1), 323-334.

6. Battochio, R. C., Stambulova, N., \& Schinke, R. J. (2016). Stages and demands in the careers of Canadian National Hockey League players. Journal of sports sciences, 34(3), 278-288.

7. Brandão, M.R.F., \& Vieira, L.F. (2013). Athletes' careers in Brazil: Research and application in the land of ginga. In N. Stambulova, y T.V. Ryba (Eds.), Athletes' careers across cultures (pp. 43-52). London: Routledge.

8. Chambers, T., Gordon, S., \& Morris, T. (2013). Athletes'careers in Australia: From retirement to education andbeyond. In Stambulova, N. B. y Ryba, T. V. (Eds.). Athletes' careers across cultures (pp. 17-30). London: Routledge.

9. Cecić Erpič, S., Wylleman, P., \& Zupančič, M. (2004). The effect of athletic and non-athletic factors on the sports career termination pro- 
cess. Psychology of Sport and Exercise, 5(1), 45-59.

10. Conzelmann, A. y Nagel, S. (2003). Professional careers of the German Olympic athletes. International review for the sociology of sport, 38(3), 259-280.

11. Cohen J. (1988). Statistical power analysis for the behavioural sciences. Hillsdale: L. Erlbaum Associates.

12. Cosh, S.y Tully, P.J. (2014). "All I have to do is pass": A discursive analysis of student athletes' talk about prioritising sport to the detriment of education to overcome stressors encountered in combining elite sport and tertiary education. Psychology of Sport and Exercise, 15(2), 180-189.

13. Fortunato, V., \& Marchant, D. (1999). Forced retirementfrom elite football in Australia. Journal of Personal and Interpersonal Loss, 4, 269 280 .

14. Gamero, E. (2012). Fundamentos de derecho deportivo:(adaptado a estudios no jurídicos).Madrid: Tecnos.

15. Henry, I. (2013). Athlete development, athlete rights and athlete welfare: A European Union perspective. The International Journal of the History of Sport, 30(4), 356-373.

16. Hickey, C., \& Kelly, P. (2008). Preparing not to be a footballer: Higher education and professional sport. Sport, Education and Society, 13,477494. DOI: $10.1080 / 13573320802445132$

17. Lavallee, D., \& Robinson, H. (2007). In pursuit of an identity: A qualitative exploration of retirement from women's artistic gymnastics. Psychology of Sport and Exercise, 8(1), 119-141.

18. North, J., \&Lavallee, D. (2004). An investigation of potential users of career transition services in the United Kingdom. Psychology of Sport and Exercise, 5, 77-84.DOI: 10.1016/S1469-0292(02)00051-1.

19. Park, S., Lavallee, D., \& Tod, D. (2013). Athletes' career transition out of sport: A systematic review. International Review of Sport and Exercise Psychology, 6(1), 22-53. http://dx.doi.org/10.1080/175098 4X.2012.687053

20. Pink, M., Saunders, J., \& Stynes, J. (2015) Reconciling the maintenance of on-field success with off-field player development: A case study of a club culture within the Australian Football League. Psychology of Sport and Exercise, 21, 98-108.

21. Price, N., Morrison, N., \& Arnold, S. (2010). Life out of the limelight: Understanding the non-sporting pursuits of elite athletes. International Journal of Sport and Society, 1(3), 69-79.

22. Ryan, M.J., \& Chambers, T.P. (2015) An exploration of career decisionmaking in the Australian Football League: Does playing experience matter?. Australian Journal of Career Development, 24(2) 114-119.

23. Roberts, C. M.; Mullen, R.; Evans L., \& Hall R. (2015) An in-depth ap- praisal of career termination experiences in professional cricket, Journalof Sports Sciences, 33 (9), 935-944, DOI: 10.1080/02640414.2014.977936

24. Samuel, R. D., \& Tenenbaum, G. (2011). The role of changing athlete's careers: A scheme of change for sport psychology practice. The Sport Psychologist, 25, 233-252.

25. Stambulova, N. (2010). Counselling athletes in career transitions: The five-step career planning strategy. Journal of Sport Psychology in Action, 1(2), 95-105.

26. Stambulova, N., Alfermann, D., Statler, T., \&Côté, J. (2009). ISSP position stand: Career development and transitions of athletes. International journal of sport and exercise psychology, 7(4), 395-412.

27. Taylor, J., \& Ogilvie, B. C. (1994). A conceptual model of adaptation to retirement among athletes. Journal of Applied Sport Psychology, 6(1), 1-20. http://dx.doi.org/10.1080/10413209408406462

28. Taylor, J., Ogilvie, B., \& Lavallee, D. (2006). Career transition among athletes: Is there life after sports? In J. M. Williams (Ed.), Applied sport psychology (pp. 595-615). New York:McGraw Hill.

29. Tekavc J., Wylleman P., \&CecićErpić, S. (2015). Perceptions of dual career development among elite level swimmers and basketball players. Psychology of Sport \& Exercise, 21, 27-41.

30. Torregrosa, M., Sánchez, X., \& Cruz, J. (2004). El papel del psicólogo del deporte en el asesoramiento académico-vocacional del deportista de élite. Revista de psicología del deporte, 13(2), 215-228.

31. Torregrosa, M., Ramis, Y., Pallarés, S., Azócar, F., \& Selva, C. (2015) Olympic athletes back to retirement: a qualitative longitudinal study. Psychology of Sport \& Exercise.21, 50-56. doi: 10.1016/j.psychsport.2015.03.003

32. Van Raalte, J. L., \& Andersen, M. B. (2007). When sport psychology consulting is a means to an end (ing): Roles and agendas when helping athletes leave their sports. Sport Psychologist, 21(2), 227-242.

33. Vilanova, A., \&Puig, N. (2014). Personal strategies for managing a second career: The experiences of Spanish Olympians. International Re view for the Sociology of Sport, 42(2), 1-18.

34. Wylleman, P., \&Lavallee, D. (2004). A developmental perspective on transitions faced by athletes. In M. Weiss (Ed.), Developmental sport psychology (pp 507-527). Morgantown, WV: Fitness Information Technology.

35. Wylleman, P., Reints, A., \& De Knop, P. (2013). A developmental and holistic perspective on the athletic career. In L. Wei (Ed.), Abstracts of the ISSP 13th World Congress of Sport Psychology. 9 (p. 2). Beijing: ISSP - Beijing Sport University. 